\title{
COMMUNICATION
}

Cite this: DOI: 10.1039/x0xx00000x

Received 00th January 2012

Accepted 00th January 2012

DOI: $10.1039 / \times 0 \times x 00000 x$

www.rsc.org/

\section{A Fast, Efficient and Simple Method for the Synthesis of Cyclic Alkenyl Fluorides by a Fluorinative Carbocyclization Reaction}

\author{
Pedro Alonso, ${ }^{\mathrm{a}}$ Pilar Pardo, ${ }^{\mathrm{a}}$ Francisco J. Fañanás, ${ }^{* \mathrm{a}}$ and Félix Rodríguez ${ }^{* \mathrm{a}}$
}

\begin{abstract}
A simple transformation of alkynol or enyne derivatives into cyclic alkenyl fluorides by means of using tetrafluoroboric acid as the proton and fluoride source is reported. This study includes the first biomimetic cationic cyclization / nucleophilic fluorination reaction of polyenyne derivatives to give terpenoid derivatives.
\end{abstract}

Although fuorinated organic molecules are rarely isolated from natural sources, organofluorine compounds are exceptionally important bioactive products with an unquestionable value as pharmaceuticals, agrochemicals, new materials and Positron Emission Tomography (PET) imaging agents. ${ }^{1}$ As a consequence, the field of synthetic fluorine chemistry has experienced an incredible increase in research activity over the past few years. ${ }^{2}$ However, the establishment of new cost effective fluorination protocols by using simple fluorinating reagents remains as a challenging goal.

The introduction of fluorine into an organic molecule can be achieved by using either an electrophilic or a nucleophilic fluorinating reagent. ${ }^{1,2}$ Although important advances have been accomplished in the field of electrophilic fluorination, this strategy still requires for the use of expensive and sensitive non-conventional reagents that in most cases behave as active oxidants. Much more appealing is the use of nucleophilic fluorinating reagents using abundant and inexpensive fluoride sources. However, the main problem of this strategy is precisely the weak nucleophilic character of the fluoride anion. In an attempt to overcome the difficulties associated to the formation of C-F bonds by either of these strategies, chemists have recently developed several ingenious methods based on the use of metal mediated or catalysed processes that efficiently provide organofluorine molecules. ${ }^{3}$ However, if possible, the use of metal-based methods in late stage fluorinations should be avoided in order to facilitate the purification of the final products. Finally, it should be noted that although a great variety of methods for the synthesis of aryl- and alkyl-fluorides have been developed, a much more undeveloped field is the synthesis of alkenyl-fluorides. ${ }^{4}$ Thus, the development of new efficient methods for the synthesis alkenyl fluorides seems an area of high interest. In this context, we devised a nucleophilic fluorocyclization strategy to access to cyclic alkenyl fluorides based on a very simple concept that surprisingly has not been exploited till now (Scheme 1).

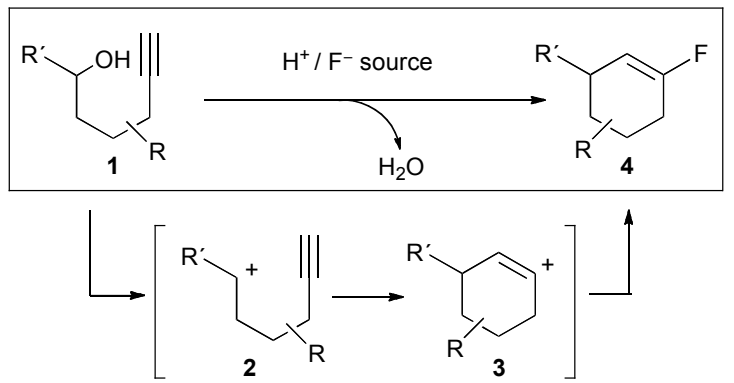

Scheme 1. Our approach to get cyclic alkenyl fluorides

Thus, we supposed that alkynyl-substituted alcohols $\mathbf{1}$ would produce the cationic species $\mathbf{2}$ under treatment with an acid. This cation could be trapped by the alkyne to deliver a new alkenyl cation 3 that in the presence of an appropriate source of fluorides should evolve to the corresponding alkenyl fluorides 4. Support to this simple strategy was found in some recent works where alkenyl cations similar to $\mathbf{3}$, but generated through other ways, were trapped by chloride (bromide) anions. ${ }^{6}$ In this context, it should be noted that, as far as we know, a general method to trap alkenyl cations with fluorides has not been reported. On the contrary, the formal trapping of aryl cations by fluorides coming from a tetrafluoroborate anion, is the basis of the well-known Balz-Schiemann synthesis of aryl fluorides. ${ }^{7}$

All the above commented facts made us to be confident about the viability of our proposal and in particular about the possibility of trapping the alkenyl cation intermediate 3 by fluoride anions if the reaction were performed in the absence of any other nucleophile. Thus, we thought that tetrafluoroboric 
acid could be the ideal reagent to perform our devised reaction because it would provide the proton necessary to generate the initial carbocation 2 and also the fluoride to trap the alkenyl cation $3 .{ }^{8}$ However, we were aware about the more than likely competing reaction of formation of a ketone derivative as recently reported by Y. Yamamoto and co-workers. ${ }^{9}$ In this process the alkenyl cation 3 is trapped by the molecule of water formed in the initial dehydration reaction of 1 . Perhaps, the occurrence of this "undesired" process is eventually the reason why our proposed reaction has not been developed. Thus, our goal was to supress the above commented hydration reaction and then we thought that an appropriate selection of the solvent of the reaction could be crucial. In this sense, solvents where the in situ formed water was as much insoluble as possible could be ideal (i.e. alkanes). In fact, to our delight, we found that hexynol derivatives 1 reacted with one equivalent of tetrafluoroboric acid diethyl ether complex $\left(\mathrm{HBF}_{4} \cdot \mathrm{Et}_{2} \mathrm{O}\right)$ in hexane as solvent at room temperature to give the corresponding cyclohexenyl fluorides 4 in very high yield (Scheme 2).
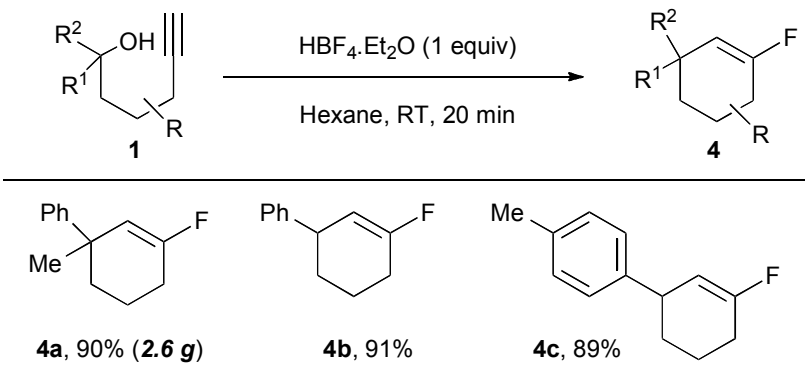<smiles>COc1ccc(C2C=C(F)CCC2)cc1</smiles><smiles>COc1cccc(CC(C)Cc2cccc(F)c2)c1</smiles><smiles>CCCCCCCCCCCC</smiles>

4d, $92 \%$

$$
\text { 4e, } 93 \%
$$

$$
\text { 4f, } 85 \%
$$<smiles>CC1(C)CC(F)=CC(C)(c2ccccc2)C1</smiles>

$4 g, 96 \%$ $\mathrm{MeO}$<smiles>COc1ccc(C2(c3ccc(OC)cc3)C=C(F)CCC2)cc1</smiles><smiles>CCC1C=C(F)Cc2ccccc21</smiles><smiles>CC1(c2ccccc2)C=C(F)Cc2ccccc21</smiles><smiles></smiles><smiles>C#CCC1(c2ccccc2)C=C(F)CCC1</smiles>

$4 n, 96 \%$<smiles>FC1=CC(c2ccccc2)c2ccccc2C1</smiles>

$4 \mathrm{~m}, 94 \%$<smiles>[N+]=[N+]([O-])C1CCC(c2ccccc2)(c2ccccc2)C=C1F</smiles>

$4 i, 90 \%^{\mathrm{a}}$



Scheme 2. Synthesis of cyclohexenyl fluorides 4a-n from alkynols 1. ${ }^{\text {a }} 8$ hours were required for completion of the reaction.
As shown, the reaction allowed the synthesis of cyclohexenyl fluorides 4 substituted at several positions. Particularly interesting are those dihydronaphthalene derivatives $4 \mathbf{k}-\mathbf{m}$ easily obtained in high yield. The reactions were very fast $(20$ minutes) for secondary and alkyl-substituted tertiary starting hexynol derivatives $\mathbf{1}$. The reaction time had to be extended only for tertiary alkynol derivatives containing two aryl groups $(4 \mathbf{h}-\mathbf{j})$. It should be noted that the reaction worked perfectly for terminal alkynes leading exclusively to the 6-endo cyclization products 4 shown. However, when internal alkynes were used the reaction lacked generality and usually no reaction or formation of mixtures of different products was observed. Moreover, we were not able to extend this methodology to the synthesis of other cycloalkenyl derivatives with a different ring size. Instead of a limitation, in some cases, this can be an advantage. Thus, when two alkynes are present in the starting material $\mathbf{1}$ a selective reaction is observed. For example, compound 4n obtained from the di-yne derivative 1n, was isolated in very high yield as a result of a cyclization involving just one of the alkynes. No competitive reaction of the other alkyne in the cyclization process was noticed and then the remaining alkyne could be used for further functionalization. Finally, we have verified that the reaction can be performed on a gram scale by easily preparing 2.6 grams of $\mathbf{4 a}$ in one batch without problems.

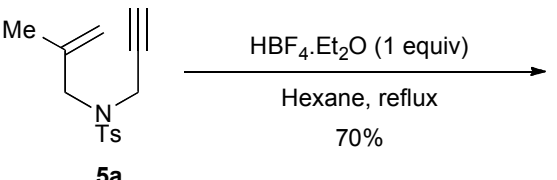

$5 a$<smiles>C#CCC(COC)(CC(=C)C)C(OC)OC</smiles>

$5 b$

Scheme 3. Cyclic alkenyl fluorides 4o,p from enynes $\mathbf{5 a , b}$
As shown in Scheme 1, the first step of this reaction is the formation the cationic species 2 . Theoretically, these cations could be accessible not only from alkynol derivatives 1 through a dehydration reaction but also from enyne derivatives through a protonation of the alkene moiety. Then, we tried our fluorination conditions on enyne derivatives 5a,b (Scheme 3). As shown, the desired fluor-containing products were obtained. These results demonstrate that interesting fluorinated heterocyclic compounds such as the tetrahydropyridine derivative 40 are also easily available through this strategy.

In an attempt to show some of the potential of our methodology we have performed some simple biomimetic polyene cyclizations (Scheme 4). Thus, when geraniol-derived dienyne $\mathbf{6 a}$ and nerol-derived dienyne $\mathbf{6 b}$ were reacted with one equivalent of $\mathrm{HBF}_{4} \cdot \mathrm{Et}_{2} \mathrm{O}$ in hexane as solvent at room temperature, the corresponding fluorinated bicyclic meroterpenes 7 were obtained in high yield after a short reaction time $(20 \mathrm{~min}) .{ }^{10}$ As far as we know, these are the first examples where a nucleophilic fluorination process is implied in a cationic biomimetic cyclization. ${ }^{11}$ 
<smiles>C#CCCC=C(C)CCC=C(C)C</smiles>
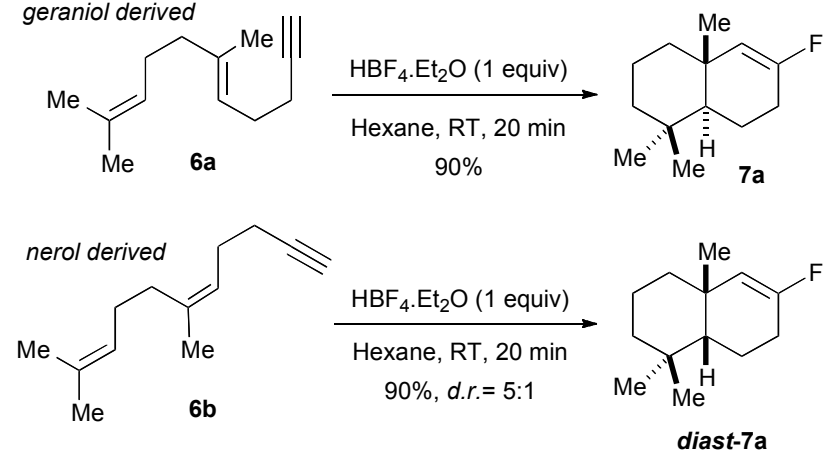

Scheme 4. Biomimetic cyclizations of polyenyne derivatives 6 to get terpenoids 7

\section{Conclusions}

In summary, we have developed a novel reaction for the synthesis of cyclic alkenyl fluorides where the source of fluoride is simply tetrafluoroboric acid. This process complements and challenges the conventional fluorination reactions. Moreover, this reaction is one of the very few examples of fluorinative cyclization reactions where the incorporation of the fluorine atom occurs by nucleophilic addition of the fluoride anion. The process, in a global sense, could be considered as a variant of the Balz-Schiemann reaction to get alkenyl fluorides instead of aryl fluorides. The reaction here described is fast, safe, cost effective, high yielding, technically very simple, scalable and it proceeds without the need of any metallic reagent or catalyst. Therefore, we anticipate its potential in the synthesis of bioactive pharmaceuticals and ${ }^{18} \mathrm{~F}$-labelled tracers. Also, we have developed an unprecedented biomimetic cationic cyclization / nucleophilic fluorination reaction to yield interesting fluorinated terpenoid derivatives. This is the first biomimetic cationic poly-cyclization / nucleophilic fluorination reaction.

We gratefully acknowledge financial support from MINECOSpain (grant CTQ2010-16790), MEC (FPU-predoctoral grant to P. A. and FPI-predoctoral grant to P. P.).

\section{Notes and references}

a Instituto Universitario de Química Organometálica "Enrique Moles", Universidad de Oviedo, Julián Clavería 8, 33006-Oviedo, Spain. Fax: (+34) 985103446; Tel: (+34) 985106224; E-mail: fjfv@uniovi.es; frodriguez@uniovi.es

1 Bioorganic and Medicinal Chemistry of Fluorine, eds. J.-P. Bégué and D. Bonnet-Delpont, Wiley, New Jersey, 2007.

2 Recent reviews: (a) T. Furuya, J. E. M. N. Klein and T. Ritter, Synthesis, 2011, 1804-1821; (b) S. Purser, P. R. Moore, S. Swallow and V. Gouverneur, Chem. Soc. Rev., 2008, 37, 320-330; (c) K. Müller, C. Faeh and F. Diederich, Science, 2007, 317, 1881-1886.

3 For two excellent reviews, see: (a) C. Hollingworth and V. Gouverneur, Chem. Commun., 2012, 48, 2929-2942; (b) T. Furuya, A. S. Kamlet and T. Ritter, Nature, 2011, 473, 470-477.
4 Most of the recent reported methods for the synthesis of alkenyl fluorides are based on electrophilic fluorination reactions promoted by metallic complexes. (a) T. Xu, X. Mu, H. Peng and G. Liu, Angew. Chem. Int. Ed., 2011, 50, 8176-8179; (b) T. De Haro and C. Nevado, Chem. Commun., 2011, 47, 248-249; (c) H. Peng and G. Liu, Org. Lett., 2011, 13, 772-775; (d) H. Zhang, C.-B. Zhou, Q.-Y. Chen, J.-C. Xiao and R. Hong, Org. Lett., 2011, 13, 560-563; (e) B. C. Gorske, C. T. Mbofana and S. J. Miller, Org. Lett., 2009, 11, 4318-4321; (f) T. Furuya and T. Ritter, Org. Lett., 2009, 11, $2860-$ 2863; (g) M. Schuler, F. Silva, C. Bobbio, A. Tessier and V. Gouverneur, Angew. Chem. Int. Ed., 2008, 47, 7927-7930; (h) J. A. Akana, K. X. Bhattacharyya, P. Müller and J. P. Sadighi, J. Am. Chem. Soc., 2007, 129, 7736-7737. For an interesting review, see: G. Landelle, M. Bergeron, M.-O. Turcotte-Savard and J.-F. Paquin, Chem. Soc. Rev., 2011, 40, 2867-2908.

5 Very few examples of "nucleophilic fluorocyclization reactions" have been reported: (a) S. Suzuki, T. Kamo, K. Fukushi, T. Hiramatsu, E. Tokunaga, T. Dohi, Y. Kita and N. Shibata, Chem. Sci., 2014, 5, 2754-2760; (b) K.-P. Wang, S. Y. Yun, P. Mamidipalli and D. Lee Chem. Sci., 2013, 4, 3205-3211. In contrast, "electrophilic fluorocyclization reactions" have been widely investigated. See for example: (c) J. R. Wolstenhulme, J. Rosenqvist, O. Lozano, J. Ilupeju, N. Wurz, K. M. Engle, G. W. Pidgeon, P. R. Moore, G. Sandford and V. Gouverneur, Angew. Chem. Int. Ed., 2013, 52, 9796-9800; (d) W. Kong, P. Feige, T. de Haro and C. Nevado Angew. Chem. Int. Ed., 2013, 52, 2469-2473; (c) V. Rauniyar, A. D. Lackner, G. L. Hamilton and F. D. Toste, Science, 2011, 334, 16811684; (e) T. Ishimaru, N. Shibata, T. Horikawa, N. Yasuda, S. Nakamura, T. Toru and M. Shiro, Angew. Chem. Int. Ed., 2008, 47, 4157-4161; (f) N. Shibata, T. Tarui, Y. Doi and K. L. Kirk, Angew. Chem. Int. Ed., 2001, 40, 4461-4463.

6 (a) M.-A. Beaulieu, K. C. Guérard, G. Maertens, C. Sabot and S. Canesi, J. Org. Chem., 2011, 76, 9460-9471; (b) P. O. Miranda, R. M. Carballo, V. S. Martín and J. I. Padrón, Org. Lett., 2009, 11, $357-$ 360; (c) J. Sun and S. A. Kozmin, J. Am. Chem. Soc., 2005, 127, 13512-13513; (d) A. Balog, S. J. Geib and D. P. Curran, J. Org. Chem., 1995, 60, 345-352.

7 G. Balz and G. Schiemann, Ber. Dtsch. Chem. Ges., 1927, 60, 11861190 .

8 For a recent work where $\mathrm{HBF}_{4}$ is used for the fluorination of $\beta$ ketoesters, see: R. Pasceri, H. E. Bartrum, C. J. Hayes and C. J. Moody, Chem. Commun., 2012, 48, 12077-12079.

9 T. Jin, M. Himuro and Y. Yamamoto, Angew. Chem. Int. Ed., 2009, 48, 5893-5896. See also: T. Jin, M. Himuro and Y. Yamamoto, J. Am. Chem. Soc., 2009, 132, 5590-5591.

10 Compounds 7 could be considered as drimane-type sesquiterpenes. Natural terpenes and unnatural analogues from these families have shown a wide spectrum of useful biological activities. See: (a) K. Kobayashi, N. Masako, M. Fukushima, Y. Shiobara and M. Kodama, Agric. Biol. Chem., 1988, 52, 77-83; (b) A. Ulubelen, S. Öksü, U. Kolak, H. Birman and W. Voelter, Planta Med., 2000, 66, 627-629; (c) B. J. M. Jansen and A. de Groot, Nat. Prod. Rep., 2004, 21, 449477; (d) C.-L. Lee, L.-C. Chiang, L.-H. Cheng, C.-C. Liaw, M. H. A. El-Razek, F.-R. Chang and Y.-C. Wu, J. Nat. Prod., 2009, 72, 15681572; (e) A. Abad, C. Agulló, A. C. Cuñat, A. González-Coloma and D. Pardo, Eur. J. Org. Chem., 2010, 2182-2198; (f) M. Fronza, R. 
Murillo, S. Ślusarczyk, M. Adams, M. Hamburger, B. Heinzmann, S Laufer and I. Merfort, Bioorg. Med. Chem., 2011, 19, 4876-4881.

11 As far as we know, biomimetic cationic cyclization / nucleophilic fluorination reactions have not been reported. However, an excellent work on the alternative biomimetic cyclization / electrophilic fluorination reaction of polyenes has been recently reported: N. A. Cochrane, H. Nguyen and M. R. Gagne, J. Am. Chem. Soc., 2013, 135, 628-631. 


\section{COMMUNICATION}

\section{Table of Contents}

Tetrafluoroboric acid promotes a fluorinative carbocyclization reaction to give cyclic alkenyl fluorides in a process where the incorporation of the fluorine atom occurs through the nucleophilic addition of the fluoride anion.

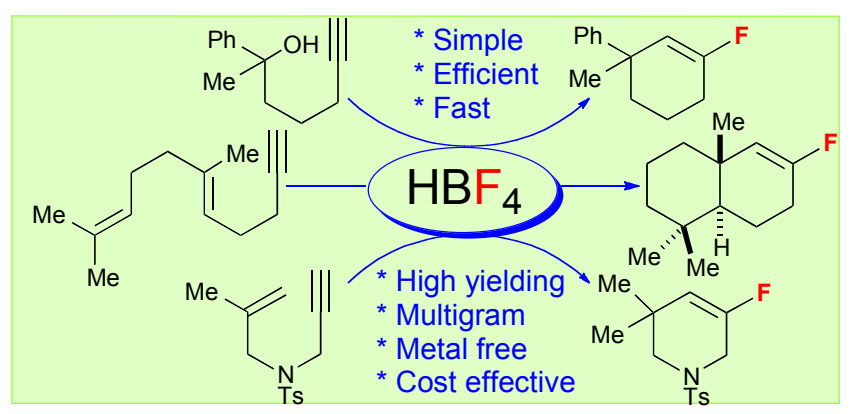

